\title{
Insegurança alimentar, consumo alimentar e estado nutricional de mulheres beneficiadas pelo Programa Bolsa Família
}

\author{
Food insecurity, consumption and nutritional status of women benefited by the \\ Bolsa Família program
}

\author{
Jaquieli Gruhm Franco (D)1, Marilene Cassel Bueno (D)2, Vanessa Ramos Kirsten (D), \\ Greisse Viero da Silva Leal (1) 1
}

Universidade Federal de Santa Maria (UFSM), Campus Palmeira das Missões, Palmeira das Missões, RS, Brasil.

${ }^{2}$ Universidade Federal do Rio Grande do Sul (UFRGS), Porto Alegre, RS, Brasil.

RESUMO

Objetivo: O objetivo deste estudo foi avaliar a prevalência de insegurança alimentar em mulheres beneficiadas pelo Programa Bolsa Família, relacionando com o consumo alimentar, estado nutricional e a classe socioeconômica. Materiais e Métodos: Estudo transversal com 201 mulheres em Palmeira das Missões-RS. A insegurança alimentar foi avaliada pela Escala Brasileira de Insegurança Alimentar. O consumo alimentar pelos marcadores de consumo alimentar, a classe socioeconômica pelo Critério de Classificação Econômica Brasil e o estado nutricional pelo índice de massa corporal.

Resultados: A média de idade foi de 37,92 (desvio padrão 10,39 anos). A amostra apresentou 91,5\% de insegurança alimentar, $61,1 \%$ de excesso de peso e $75,1 \%$ eram de baixa classe socioeconômica. Foi observado alto consumo de bebidas adoçadas $(67,2 \%)$, de legumes/verduras $(74,6 \%)$ e de feijão $(77,4 \%)$. A maior prevalência de insegurança alimentar está nas classes D-E e os níveis de insegurança alimentar diminuem conforme aumenta a classe socioeconômica $(p=0,009)$. A segurança alimentar apresentou relação com o consumo de frutas e o hábito de realizar café da manhã. O sobrepeso apresentou maior prevalência na insegurança alimentar moderada $(47,4 \%)$. Conclusão: As mulheres do Programa Bolsa Família apresentaram alta insegurança alimentar relacionada ao excesso de peso.

Palavras-chave: políticas públicas; segurança alimentar e nutricional; desigualdades em saúde; mulheres.

Objective: The objective of this study was to evaluate the prevalence of food insecurity in women benefiting from the Bolsa Família program, relating to food consumption, nutritional status and socioeconomic status.

Materials and Methods: Cross-sectional study with 201 women. Food insecurity was evaluated by the Brazilian Food Insecurity Scale. Food consumption by the food consumption markers, the socioeconomic class by the Brazilian Economic Classification Criteria and the nutritional status by the body mass index.

Results: The mean age was $37.92 \pm 10.39$ years. The sample presented $91.5 \%$ of food insecurity, $61.1 \%$ of excess weight and $75.1 \%$ were of low socioeconomic class. A high consumption of sweetened beverages (67.2\%), vegetables/greens (74.6\%) and beans (77.4\%) was observed. The highest prevalence of food insecurity are in the $D$-E classes and levels of food insecurity decrease as the socioeconomic class increases $(p=0.009)$. Food security was related to fruit consumption and the habit of having breakfast. Overweight had a higher prevalence in moderate food insecurity $(47.4 \%)$.

Conclusions: Women of the Bolsa Familia program presented high food insecurity related to overweight.

Keywords: public policies; food and nutrition security; health inequalities women.

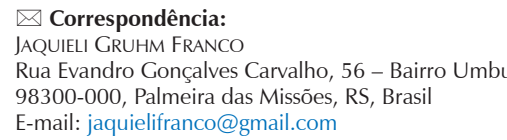




\section{INTRODUÇÃO}

Com a preocupação crescente sobre a segurança alimentar e nutricional (SAN), o Brasil obteve destaque em âmbito internacional como referência para políticas públicas de alimentação e nutrição, com a criação do Programa Fome Zero ${ }^{1}$, o qual, objetivou a superação da fome e miséria, fornecendo incentivos à agricultura familiar, à alimentação escolar e à transferência de renda. Em 2006, a Lei Orgânica de Segurança Alimentar e Nutricional LOSAN( Lei no 11.346/2006)² é instituída, propondo um sistema nacional similar ao Sistema Único de Saúde (SUS), com os princípios da universalidade, participação social, intersetorialidade e equidade, que define SAN como a execução do direito de todos os brasileiros ao acesso regular e permanente a alimentos de qualidade e em quantidade suficiente, respeitando a diversidade cultural da população, sem comprometer o acesso às outras necessidades básicas.

Em 2015, a Organização das Nações Unidas ${ }^{3}$ (ONU) apontou que o Brasil conquistou as metas estabelecidas nos Objetivos do Desenvolvimento do Milênio, saindo do Mapa da Fome. Esta conquista tornou-se consistente devido às políticas públicas que abordam a SAN de modo intersetorial, com produção e disponibilidade de alimentos, melhores condições de vida, acesso a alimentos e água de qualidade e a serviços de saúde e educação. Diversos os programas responsáveis por esta conquista, entre eles: o Programa de Aquisição de Alimentos (PAA), do qual, prevê a compra de produtos alimentícios diretamente da agricultura familiar para abastecer programas e populações em condições de Insegurança Alimentar (IA); a ampliação do Programa Nacional de Alimentação Escolar (PNAE) e a consolidação de Programas de Transferência Condicionada de Renda (PTCR) 1 . A Organização das Nações Unidas para a Alimentação e a Agricultura $(\mathrm{FAO})^{3}$ declarou ainda que, a proteção social, com a promoção ao acesso à renda, assistência nutricional, saúde e melhor educação, contribuiu diretamente na redução da fome e da desnutrição.

O Programa Bolsa Família (PBF) possui a missão de diminuir as desigualdades sociais e proporcionar aos seus participantes acesso às redes públicas de saúde, educação e assistência social, por meio da transferência condicionada de renda, combatendo a pobreza e promovendo a SAN 4,5 . A transferência condicionada de renda, vem apresentando melhorias significativas no Brasil, principalmente ao que tange à insegurança alimentar, visto que se apresenta comumente em famílias que se encontram no contexto de vulnerabilidade social ${ }^{6}$.

$\mathrm{O}$ PBF proporciona às famílias, principalmente para mulheres, a autonomia de maior independência financeira, aumentando o poder de compra. As mulheres são 94,5\% das vezes as titulares do cartão do benefício, além de serem responsáveis e conhecerem melhor as necessidades de sua família ${ }^{7}$, são mais susceptíveis a sentir os reflexos da situação de insegurança alimentar. Estudos $7,8,9$ retratam que a maior parte dos beneficiários gastam o dinheiro recebido com a alimentação, porém nem sempre, esses alimentos possibilitam qualidade nutricional, pois estas famílias acabam a dar preferência a alimentos de alta densidade energética, de baixo valor nutricional, e o consumo de produtos industrializados.

Pesquisas referentes à situação de insegurança alimentar com beneficiários do $\mathrm{PBF}^{10,11,12}$, apresentam em sua maioria alta prevalência de IA, chefes de família com baixa escolaridade e baixo poder aquisitivo, e muitas vezes com foco no estado nutricional de crianças. Até o momento são poucos os estudos que avaliam o consumo alimentar, estado nutricional e relacionam com a SA das mulheres beneficiárias. Avaliações de programas de transferência de renda possibilitam identificar os limites e desafios a serem desconstruídos pelos formuladores, gestores e implementadores destes programas. Desta forma, o presente estudo teve como objetivo avaliar a prevalência de insegurança alimentar em mulheres beneficiadas pelo Programa Bolsa Família em um município no interior do Rio Grande do Sul, e relacionar com o consumo alimentar, estado nutricional e a situação socioeconômica das mesmas.

\section{MATERIAIS E MÉTODOS}

A pesquisa, de delineamento transversal, foi realizada com mulheres titulares beneficiadas pelo Programa Bolsa Família, residentes no município de Palmeira das MissõesRS no segundo semestre de 2016.

Palmeira das Missões está localizada na região norte do estado do Rio Grande do Sul, distante da capital gaúcha, Porto Alegre, em cerca de $372 \mathrm{~km}$. É um município com grande concentração de latifúndio ${ }^{13}$, visto que a área municipal utilizada para o cultivo da soja, no período de 2005 a 2010, foi de 63,69\%, em média. Segundo o último censo demográfico do Instituto Brasileiro de Geografia e Estatística $\left(\right.$ IBGE) ${ }^{14}$, realizado em 2010, a população estimada do município era de 34.328 habitantes, dos quais, 29,831 (86,3\%) residem na zona urbana. A área da unidade territorial em 2015 era de 1.419,430 km². No mesmo ano de realização do Censo, o índice de desenvolvimento humano (IDH) do município foi de 0,737.

Palmeira das Missões beneficiou durante o mês de agosto de 2016 cerca de 1783 famílias pelo PBF ${ }^{15}$, representando uma cobertura de $83 \%$ da estimativa de famílias pobres no 
município. Levando em consideração o número de famílias beneficiadas em 2016 e assumindo uma margem de erro de $5 \%$ para um intervalo de confiança de $95 \%$, com uma estimativa máxima de segurança alimentar a ser encontrada de $17 \%{ }^{7}$, resultou em uma amostra calculada mínima de 194 mulheres.

A coleta de dados ocorreu em todos os dias referentes a pesagem das famílias acolhidas pelo Programa. A amostra pertence a 29 diferentes localidades, contemplando todas as áreas do município. Os locais designados para a coleta dos dados foram escolas, Estratégias Saúde da Família, pavilhões e igrejas da comunidade. As mulheres deslocaram-se até o local com a finalidade do cumprimento da condicionalidade de acompanhamento do estado nutricional. No local da pesagem, as mulheres foram convidadas a participar da pesquisa.

A variável dependente foi constituída pelo diagnóstico de insegurança alimentar por meio do instrumento da Escala Brasileira de Insegurança Alimentar (EBIA $)^{16}$, este composto por 14 questões, direcionadas aos 3 últimos meses, com opções de "Sim" e "Não", cada resposta afirmativa, corresponde a um ponto. A EBIA nivela os domicílios, permitindo estimar as prevalências e classificá-los em quatro diferentes níveis de segurança ou insegurança alimentar. A soma dos pontos para famílias constituídas com ao menos um menor de 18 anos é diferente daquelas usadas para classificar domicílios que residem apenas adultos, pois esses, são utilizadas apenas oito das 14 questões.

As categorias foram classificadas em segurança alimentar com pontuação 0 , tanto para famílias com menores de 18 anos, como para as que possuem somente adultos. Foi considerado insegurança alimentar leve, pontuação de 1 a 5 para famílias com menores de 18 anos e 1 a 3 as famílias sem menores de 18 anos, na qual há preocupação com a falta de alimentos no futuro próximo, retratando um componente psicológico de insegurança, também há situação de comprometimento na qualidade da alimentação. Insegurança alimentar moderada, considerou-se pontuação que varia de 6 a 9 para famílias com menores de 18 anos e 4 a 5 sem menores de 18 anos, na qual, a família apresenta restrição qualitativa e quantitativa na dieta, resultante na falta de alimentos entre os adultos. Insegurança alimentar grave, com pontuação de 10 a 14 famílias com menores de 18 anos e 6 a 8 pontos em famílias sem menores de 18 anos, onde a condição em que há deficiência quantitativa de alimento, levando à situação de fome ${ }^{17}$.

Em relação ao consumo alimentar, foi aplicado o formulário do Sistema de Vigilância Alimentar e Nutricional $\left(\right.$ SISVAN) ${ }^{18}$, utilizando somente o bloco de questões para maiores de 2 anos, adolescentes, adultos, idosos e gestantes.
O formulário é composto pela avaliação de alimentos consumidos no dia anterior, além de identificar a quantidade de refeições realizadas ao longo do dia, também o hábito de assistir televisão durante as refeições. A utilização do formulário seguiu as recomendações e instrumentos padronizados pelo Ministério da Saúde ${ }^{18}$.

$\mathrm{Na}$ obtenção dos indicadores socioeconômicos e demográficos, utilizaram-se variáveis de estado civil, idade, número de moradores no domicílio, se a moradia possuía morador menor de 18 anos. Para conhecimento da classe socioeconômica, foi aplicado o Critério de Classificação Econômica Brasil (CCEB) validado em 2015, disponibilizado pela Associação Brasileira de Empresas de Pesquisa (ABEP) ${ }^{19}$, que, classifica os indivíduos segundo seu poder de compra, além de fornecer o número de moradores no domicílio. O sistema de pontos é contabilizado através de variáveis da quantidade de bens, grau de instrução do chefe da família e acesso a serviços públicos, onde cada resposta terá seu respectivo valor em pontos. Para a classificação dos resultados foi utilizado o critério de avaliação de pontos, onde a divisão definida resulta exclusivamente as classes econômicas (A, B1, B2, C1, C2, D-E).

Para avaliação do estado nutricional, aferiu-se o peso e altura para análise do Índice de Massa Corporal (IMC), valores coletados em conjunto com Agentes Comunitárias de Saúde (ACS) do município. Para os procedimentos de diagnóstico nutricional segue o documento de Norma Técnica do Sistema de Vigilância Alimentar e Nutricional (SISVAN) ${ }^{20}$, que recomenda o uso da classificação do IMC proposta pela Organização Mundial de Saúde (OMS). Mulheres gestantes tiveram seu estado nutricional excluído.

O banco de dados foi organizado e digitado no Microsoft Office Excel 2013 e posteriormente analisado no software SPSS (Statistical Package for the Social Sciences) versão 18.0. Os dados foram analisados por meio de estatística descritiva simples (média, desvio padrão e percentual) e para a análise de comparações da IA com as variáveis de consumo alimentar, estado nutricional e classe socioeconômica, foi utilizado o teste qui-quadrado. Nestas análises foram aplicados teste post-hoc, com ajuste por Bonferroni. Diferenças estatisticamente significativas foram consideradas quando $p \leq 0,05$.

A participação das mulheres no estudo foi condicionada à assinatura do termo de consentimento livre e esclarecido. Este estudo foi aprovado pelo Comitê de Ética em Pesquisa da Universidade Federal de Santa Maria sob o número de CAAE 60544216.0.0000.5346, parecer 1.812.495, e obedeceu a todos os aspectos éticos e legais para pesquisas envolvendo seres humanos de acordo com a Resolução no 466/2012 do Conselho Nacional de Saúde. 


\section{RESULTADOS}

A amostra estudada foi de 201 mulheres, com média idade de 37,92 $\pm 10,39$ anos. Das mulheres avaliadas observou-se que $43,3 \%$ eram solteiras, apresentavam baixa escolaridade $35,8 \%$ eram analfabetas ou não haviam completado o fundamental I), excesso de peso (sobrepeso + obesidade $=64,06 \%$, não eram gestantes $(96,02 \%$ ), residiam com menores de 18 anos (94,5\%) e 49\% delas recebiam o benefício há mais de 7 anos. As beneficiárias apresentam em sua maioria nível socioeconômica D-E (75,1\%), em 56,2\% dos domicílios residiam 4 ou mais pessoas. Quanto às condições de saneamento, 92,0\% estavam ligadas à rede pública de distribuição de água. A aplicação da EBIA demonstrou alta prevalência de insegurança alimentar (91,5\%), na qual 45,8\% de insegurança leve, $29,9 \%$ de moderada e $15,9 \%$ grave (Tabela 1).

De acordo com o consumo alimentar das mulheres avaliadas, observou-se que o consumo de bebidas adoçadas $(67,2 \%)$, legumes/verduras $(74,6 \%)$, e feijão $(77,4 \%)$ tiveram as maiores prevalências de consumo diário. A segurança alimentar foi relacionada com o hábito de consumir café da manhã $(p=0,048)$ e frutas $(p=0,045)$. As demais variáveis do consumo alimentar não apresentaram relação com a presença da insegurança alimentar (Tabela 2).

Quando avaliada a insegurança alimentar de acordo com o estado nutricional, observa-se que a maior prevalência de eutrofia $(53,3 \%)$ foi encontrada nas mulheres com insegurança alimentar grave. O sobrepeso apresentou percentuais elevados em todos os níveis de insegurança alimentar, porém apresenta maior prevalência na insegurança alimentar moderada $(47,4 \%)$. É possível observar que a condição de magreza não foi encontrada em mulheres em situação de segurança alimentar, bem como os valores de sobrepeso e obesidade são menores em mulheres com insegurança alimentar grave quando compara as demais classificações (Tabela 3).
Tabela 1. Caracterização socioeconômica, demográfica e insegurança alimentar de mulheres participantes do Programa Bolsa Família $(n=201)$.

\begin{tabular}{|c|c|c|}
\hline Variáveis & $\mathbf{n}$ & $\%$ \\
\hline \multicolumn{3}{|l|}{ Estado civil } \\
\hline Casada & 62 & 30,8 \\
\hline Solteira & 87 & 43,3 \\
\hline União estável & 36 & 17,9 \\
\hline Divorciada & 13 & 6,5 \\
\hline Viúva & 3 & 1,5 \\
\hline \multicolumn{3}{|l|}{ Estado nutricional* } \\
\hline Magreza & 8 & 4,17 \\
\hline Eutrofia & 61 & 31,77 \\
\hline Sobrepeso & 61 & 31,77 \\
\hline Obesidade & 62 & 32,29 \\
\hline Gestante & 8 & 3,98 \\
\hline \multicolumn{3}{|l|}{ Escolaridade da mulher } \\
\hline Analfabeto/Fundamental I incompleto & 72 & 35,8 \\
\hline Fundamental I completo/Fundamental II incompleto & 59 & 29,4 \\
\hline Fundamental completo II/Médio incompleto & 45 & 22,4 \\
\hline Médio completo/Superior incompleto & 25 & 12,4 \\
\hline \multicolumn{3}{|l|}{ Tempo de recebimento do benefício** } \\
\hline Menos de 1 ano & 26 & 13,0 \\
\hline 1 a 7 anos & 76 & 38,0 \\
\hline Mais de 7 anos & 98 & 49,0 \\
\hline Reside com menor de 18 anos & 190 & 94,5 \\
\hline \multicolumn{3}{|l|}{ Número de moradores no domicílio } \\
\hline Até 3 pessoas & 88 & 43,8 \\
\hline 4 ou mais pessoas & 113 & 56,2 \\
\hline Rua do domicilio pavimentada & 152 & 75,6 \\
\hline Abastecimento de água com rede pública & 185 & 92,0 \\
\hline \multicolumn{3}{|l|}{ Classe socioeconômico } \\
\hline B2 & 2 & 1,0 \\
\hline $\mathrm{C} 1$ & 10 & 5,0 \\
\hline $\mathrm{C} 2$ & 38 & 18,9 \\
\hline D-E & 151 & 75,1 \\
\hline \multicolumn{3}{|l|}{ Níveis de insegurança alimentar } \\
\hline Segurança alimentar & 17 & 8,5 \\
\hline Insegurança alimentar & 184 & 91,5 \\
\hline Insegurança alimentar leve & 92 & 45,8 \\
\hline Insegurança alimentar moderada & 60 & 29,9 \\
\hline Insegurança alimentar grave & 32 & 15,9 \\
\hline Total & 201 & 100 \\
\hline
\end{tabular}

* Não foi avaliado o estado nutricional de 9 mulheres.

** Uma mulher não soube referir o tempo de recebimento do benefício.

Tabela 2. Prevalências de Insegurança alimentar, de acordo com os marcadores do consumo alimentar de mulheres participantes do Programa Bolsa Família $(n=201)$.

\begin{tabular}{|c|c|c|c|c|c|c|c|}
\hline Marcadores do consumo alimentar & & $\begin{array}{c}\text { Segurança } \\
\text { alimentar } \\
\text { n (\%) }\end{array}$ & $\begin{array}{c}\text { Insegurança } \\
\text { leve } \\
\text { n (\%) }\end{array}$ & $\begin{array}{c}\text { Insegurança } \\
\text { moderada } \\
n(\%)\end{array}$ & $\begin{array}{c}\text { Insegurança } \\
\text { grave } \\
\text { n (\%) }\end{array}$ & $\begin{array}{l}\text { Total } \\
\text { n (\%) }\end{array}$ & $\begin{array}{l}\text { Valor } \\
\text { de } p\end{array}$ \\
\hline \multirow[t]{2}{*}{ Costuma realizar refeições assistindo televisão } & Sim & $6(35,3)$ & $35(38,0)$ & $28(46,7)$ & $17(53,1)$ & $86(42,8)$ & 0,391 \\
\hline & Não & $11(64,7)$ & $57(62,0)$ & $32(53,3)$ & $15(46,9)$ & $115(57,2)$ & \\
\hline \multirow[t]{2}{*}{ Costuma realizar o café da manhã } & Sim & $16(94,1)$ & $64(69,6)$ & $36(60,0)$ & $24(75,0)$ & $140(69,7)$ & 0,048 \\
\hline & Não & $1(5,9)$ & $28(30,4)$ & $24(40,0)$ & $8(25,0)$ & $61(30,3)$ & \\
\hline \multirow[t]{2}{*}{ Costuma realizar o lanche da manhã } & Sim & $2(11,8)$ & $11(12,0)$ & $7(11,7)$ & $4(12,5)$ & $24(11,9)$ & 1,000 \\
\hline & Não & $15(88,2)$ & $81(88,0)$ & $53(88,3)$ & $28(87,5)$ & $177(88,1)$ & \\
\hline \multirow[t]{2}{*}{ Costuma realizar o almoço } & Sim & $17(100,0)$ & $90(97,8)$ & $60(100,0)$ & $30(93,8)$ & $197(98,0)$ & 0,207 \\
\hline & Não & $0(0,00)$ & $2(2,2)$ & $0(0,0)$ & $2(6,3)$ & $4(2,0)$ & \\
\hline
\end{tabular}


Tabela 2 (continuação)

\begin{tabular}{|c|c|c|c|c|c|c|c|}
\hline Marcadores do consumo alimentar & & $\begin{array}{c}\text { Segurança } \\
\text { alimentar } \\
\mathbf{n}(\%)\end{array}$ & $\begin{array}{c}\text { Insegurança } \\
\text { leve } \\
\text { n (\%) }\end{array}$ & $\begin{array}{c}\text { Insegurança } \\
\text { moderada } \\
\text { n (\%) }\end{array}$ & $\begin{array}{l}\text { Insegurança } \\
\text { grave } \\
\text { n (\%) }\end{array}$ & $\begin{array}{l}\text { Total } \\
\text { n (\%) }\end{array}$ & $\begin{array}{l}\text { Valor } \\
\text { de } p\end{array}$ \\
\hline Costuma realizar o lanche da tarde & $\begin{array}{l}\text { Sim } \\
\text { Não }\end{array}$ & $\begin{array}{c}13(76,5) \\
4(23,5)\end{array}$ & $\begin{array}{l}55(59,8) \\
37(40,2)\end{array}$ & $\begin{array}{l}35(58,3) \\
25(41,7)\end{array}$ & $\begin{array}{l}20(62,5) \\
12(37,5)\end{array}$ & $\begin{array}{c}123(61,2) \\
78(38,8)\end{array}$ & 0,577 \\
\hline Costuma realizar o jantar & $\begin{array}{l}\text { Sim } \\
\text { Não }\end{array}$ & 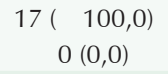 & $\begin{array}{c}86(93,5) \\
6(6,5)\end{array}$ & $\begin{array}{c}54(90,0) \\
6(10,0)\end{array}$ & $\begin{array}{c}32(100,0) \\
0(0,0)\end{array}$ & $\begin{array}{c}189(94,0) \\
12(6,0)\end{array}$ & 0,180 \\
\hline Costuma realizar a ceia & $\begin{array}{l}\text { Sim } \\
\text { Não }\end{array}$ & $\begin{array}{c}0(0,0) \\
17(100,0)\end{array}$ & $\begin{array}{c}6(6,5) \\
86(93,5)\end{array}$ & $\begin{array}{c}2(3,3) \\
58(96,7)\end{array}$ & $\begin{array}{c}1(3,1) \\
31(96,9)\end{array}$ & $\begin{array}{c}9(4,5) \\
192(95,5)\end{array}$ & 0,569 \\
\hline Consumiu feijão ontem & $\begin{array}{l}\text { Sim } \\
\text { Não }\end{array}$ & $\begin{array}{c}13(76,5) \\
4(23,5)\end{array}$ & $\begin{array}{l}74(80,4) \\
18(19,6)\end{array}$ & $\begin{array}{l}45(75,0) \\
15(25,0)\end{array}$ & $\begin{array}{c}24(75,0) \\
8(25,0)\end{array}$ & $\begin{array}{c}156(77,6) \\
45(22,4)\end{array}$ & 0,850 \\
\hline Consumiu frutas frescas ontem & $\begin{array}{l}\text { Sim } \\
\text { Não }\end{array}$ & $\begin{array}{c}11(64,7) \\
6(35,3)\end{array}$ & $\begin{array}{l}48(52,2) \\
44(47,8)\end{array}$ & $\begin{array}{l}23(38,3) \\
37(61,7)\end{array}$ & $\begin{array}{l}10(31,3) \\
22(68,8)\end{array}$ & $\begin{array}{c}92(45,8) \\
109(54,2)\end{array}$ & 0,045 \\
\hline Consumiu verduras e/ou legumes ontem & $\begin{array}{l}\text { Sim } \\
\text { Não }\end{array}$ & $\begin{array}{c}14(82,4) \\
3(17,6)\end{array}$ & $\begin{array}{l}73(79,3) \\
19(20,7)\end{array}$ & $\begin{array}{l}43(71,7) \\
17(28,3)\end{array}$ & $\begin{array}{l}20(62,5) \\
12(37,5)\end{array}$ & $\begin{array}{c}150(74,6) \\
51(25,4)\end{array}$ & 0,223 \\
\hline Consumiu hambúrguer e/ou embutidos ontem & $\begin{array}{c}\text { Sim } \\
\text { Não } \\
\text { Não sabe }\end{array}$ & $\begin{array}{c}9(52,9) \\
8(47,1) \\
0(0,0)\end{array}$ & $\begin{array}{c}32(34,8) \\
59(64,1) \\
1(1,1)\end{array}$ & $\begin{array}{c}23(38,3) \\
37(61,7) \\
0(0,0)\end{array}$ & $\begin{array}{c}6(18,8) \\
26(81,3) \\
0(0,0)\end{array}$ & $\begin{array}{c}70(34,8) \\
130(64,7) \\
1(0,5)\end{array}$ & 0,266 \\
\hline Consumiu bebida industrializada ontem & $\begin{array}{l}\text { Sim } \\
\text { Não }\end{array}$ & $\begin{array}{c}12(70,6) \\
5(29,4)\end{array}$ & $\begin{array}{l}65(70,7) \\
27(29,3)\end{array}$ & $\begin{array}{l}37(61,7) \\
23(38,3)\end{array}$ & $\begin{array}{l}21(65,6) \\
11(34,4)\end{array}$ & $\begin{array}{c}135(67,2) \\
66(32,8)\end{array}$ & 0,693 \\
\hline $\begin{array}{l}\text { Consumiu macarrão instantâneo, salgadinhos de } \\
\text { pacote ou biscoitos salgados ontem }\end{array}$ & $\begin{array}{l}\text { Sim } \\
\text { Não }\end{array}$ & $\begin{array}{c}2(11,8) \\
15(88,2)\end{array}$ & $\begin{array}{l}12(13,0) \\
80(87,0)\end{array}$ & $\begin{array}{c}7(11,7) \\
53(88,3)\end{array}$ & $\begin{array}{c}2(6,3) \\
30(93,8)\end{array}$ & $\begin{array}{c}23(11,4) \\
178(88,6)\end{array}$ & 0,780 \\
\hline $\begin{array}{l}\text { Consumiu biscoito recheado, doces ou } \\
\text { guloseimas ontem }\end{array}$ & $\begin{array}{l}\text { Sim } \\
\text { Não }\end{array}$ & $\begin{array}{c}3(17,6) \\
14(82,4)\end{array}$ & $\begin{array}{l}19(20,7) \\
73(79,3)\end{array}$ & $\begin{array}{c}8(13,3) \\
52(86,7)\end{array}$ & $\begin{array}{c}7(21,9) \\
25(78,1)\end{array}$ & $\begin{array}{c}37(18,4) \\
164(81,6)\end{array}$ & 0,659 \\
\hline
\end{tabular}

p: Teste qui-quadrado.

Tabela 3. Insegurança alimentar e sua relação com o estado nutricional de mulheres participantes do Programa Bolsa Família $\left(\mathrm{n}=192^{\circledR}\right)$.

\begin{tabular}{lcccc}
\hline & $\begin{array}{c}\text { Segurança alimentar } \\
\mathbf{n}(\%)\end{array}$ & $\begin{array}{c}\text { Insegurança leve } \\
\mathbf{n}(\%)\end{array}$ & $\begin{array}{c}\text { Insegurança moderada } \\
\mathbf{n}(\%)\end{array}$ & $\begin{array}{c}\text { Insegurança grave } \\
\mathbf{n}(\%)\end{array}$ \\
Estado nutricional & & & & Valor de $\boldsymbol{p}$ \\
Magreza & $0(0,0)$ & $5(5,6)$ & $2(3,5)$ & $1(3,3)$ \\
Eutrofia & $5(31,3)$ & $26(29,2)$ & $14(24,6)$ & $16(53,3)^{*}$ \\
Sobrepeso & $6(37,5)$ & $35(39,3)^{*}$ & $14(24,6)$ & $6(20,0)$ \\
Obesidade & $5(31,3)$ & $23(25,8)$ & $27(47,4)^{*}$ & $7(23,3)$ \\
Total & $16(100,0)$ & $89(100,0)$ & $57(100,0)$ & $30(100,0)$ \\
\hline
\end{tabular}

p: Teste qui-quadrado.

* Valores significativos quando aplicado o post-hoc para qui-quadrado.

\& Foram excluídas da avaliação nutricional 8 gestantes e uma participante com dado faltante.

Tabela 4. Prevalências de insegurança alimentar de acordo com as características socioeconômicas de mulheres participantes do Programa Bolsa Família $(n=201)$

\begin{tabular}{|c|c|c|c|c|c|}
\hline & $\begin{array}{c}\text { Segurança alimentar } \\
\text { n (\%) }\end{array}$ & $\begin{array}{c}\text { Insegurança leve } \\
n(\%)\end{array}$ & $\begin{array}{c}\text { Insegurança moderada } \\
\mathrm{n}(\%)\end{array}$ & $\begin{array}{c}\text { Insegurança grave } \\
\mathrm{n}(\%)\end{array}$ & Valor de $p$ \\
\hline \multicolumn{6}{|c|}{ Classe Socioeconômica } \\
\hline B2 & $0(0,0)$ & $2(2,2)$ & $0(, 0)$ & $0(, 0)$ & \multirow{4}{*}{0,009} \\
\hline $\mathrm{C} 1$ & $3(17,6)$ & $5(5,4)$ & $1(1,7)$ & $1(3,1)$ & \\
\hline $\mathrm{C} 2$ & $6(35,3)$ & $22(23,9)$ & $9(15,0)$ & $1(3,1)$ & \\
\hline D-E & $8(47,1)$ & $63(68,5)$ & $50(83,3)$ & $30(93,8)^{*}$ & \\
\hline
\end{tabular}

p: Teste qui-quadrado. Após aplicado post-hoc para Bonferroni, não houve diferença significativa entre as variáveis.

As maiores prevalências de insegurança alimentar estão nas classes D-E e os níveis de insegurança alimentar diminui, conforme há um aumento da classe socioeconômica $(p=0,009)$ (Tabela 4).

\section{DISCUSSÃO}

Os resultados do presente estudo demonstram que grande parte das mulheres beneficiadas são adultos jovens, solteiras 
e com baixa escolaridade. Na avaliação do estado nutricional foi possível constatar que mais da metade apresentou excesso de peso e um número expressivo apresentaram algum grau de insegurança alimentar.

A amostra estudada apresentou prevalência de insegurança alimentar superior quando comparada a pesquisas nacionais como Pesquisa Nacional de Amostras de Domicílio (PNAD) ${ }^{16}$ em 2013 (22,6\%) e a Pesquisa Nacional de Demografia e Saúde (PNDS) ${ }^{21}$ (37,5\%). Contudo, em comparação a inquéritos nacionais, esta diferença é plausível, em vista que estes estudos fazem a caracterização de famílias em diferentes classes socioeconômicas, e este avaliou uma população que já se encontra em situação de vulnerabilidade social. O Instituto Brasileiro de Análises Sociais e Econômicas (IBASE) ${ }^{7}$, encontrou em 2007, uma alta prevalência de IA $(83,1 \%)$ entre titulares do PBF. A PNDS ${ }^{21}$ também evidenciou prevalência de IA (60,9\%) em domicílios que algum morador estava inscrito em programas sociais de transferência de renda.

Estudos prévios ${ }^{8,10,11}$ demonstram altas prevalências de IA entre os beneficiários do PBF, como em Colombo-PR (81,6\%), Maceió-AL $(91,2 \%)$ e Viçosa-MG $(72,8 \%)$. Desta forma, é enfático que independente da região, esta população apresenta maior vulnerabilidade, o que justifica a necessidade do incremento na sua renda com o auxílio de programas sociais.

As prevalências de insegurança alimentar moderada $(34,1 \%)$ e grave $(20,7 \%)$ encontradas pelo IBASE$^{7}$, são próximas aos achados nesta pesquisa. O presente estudo evidencia a alta prevalência de IA leve, do qual, o estágio é caracterizado pela redução da qualidade da dieta com o propósito de manter a quantidade suficiente para as necessidades diárias ${ }^{16}$, sugerindo a escolha por alimentos mais baratos, hipercalóricos e de baixo valor nutritivo, podendo este ser o responsável pelo excesso de peso nesta população.

Titulares do PBF relataram em um estudo qualitativo ${ }^{22}$, preferência por alimentos ditos "fortes", pois estes "sustentam" o corpo. Inclusive o estudo revela o reconhecimento das famílias sobre a situação de IA retratando a dificuldade destas a adquirirem alimentos de qualidade e quantidade adequada, pois há discordância entre o preço elevado dos alimentos e a baixa renda familiar. Desta forma, revela-se a necessidade de preservar e aprimorar o Programa, consolidando com outras políticas públicas capazes de enfrentar estes problemas. O município de Palmeira das Missões atualmente não dispõe de uma política de segurança alimentar, e que talvez com a sua implementação (como por exemplo: PAA, restaurantes populares, entre outros), pudesse ser um instrumento capaz de melhorar a qualidade da dieta e os níveis de obesidade.
No cenário epidemiológico brasileiro, o consumo alimentar relaciona-se com a saúde, tornando-se um dos determinantes para o estado nutricional da população. $\mathrm{O}$ estudo inova ao utilizar o formulário recomendado pelo Ministério da Saúde ${ }^{18}$ para avaliar o consumo alimentar destas mulheres, pois pesquisas prévias ${ }^{8,9}$, avaliaram o consumo alimentar de beneficiários do PBF, com o Recordatório de 24 horas. Deve-se destacar que apesar do formulário apresentar pontos positivos, ele também apresenta algumas limitações por ser um questionário que possui questões fechadas, pode ser que algum alimento consumido pela amostra não estivesse contemplado no instrumento.

A dieta monótona, com baixo consumo de frutas e verduras, alto consumo de leguminosas, produtos industrializados, ricos em açúcares, gorduras e sódio encontrados em pesquisas ${ }^{23-27}$ são hábitos que podem estar associadas a estratégias para superar o baixo orçamento familiar, já que grande parte da renda mensal das famílias acaba por ser absorvida no custeio mensal da alimentação ${ }^{26}$. Nota-se um tripé quando se associa a obesidade, IA e pobreza, pois os três relacionados, demonstram o declínio na aquisição alimentar das famílias, tanto em quantidade como em qualidade ${ }^{8,9,24}$.

Conforme recomenda o Guia Alimentar para a População Brasileira alimentos in natura e minimamente processados devem constituir a base de alimentação, esses alimentos são predominantemente de origem vegetal e são a base para uma alimentação saudável, adequada, saborosa e promotora de um sistema alimentar sustentável. Quanto mais as pessoas forem incentivadas a buscar alimentos nos contextos locais, maior será o apoio para os produtores locais e mais a população estará próxima de um sistema alimentar justo e saudáve ${ }^{28}$. Ainda, considerando que o estudo foi realizado em um município agrícola, o incentivo ao consumo de alimentos locais, além de ir ao encontro da alimentação saudável e adequada, também pode contribuir para o desenvolvimento local.

Quanto ao estado nutricional das mulheres avaliadas, notase que o sobrepeso e a obesidade predominam semelhante a dados encontrados em outras regiões do país em adultos beneficiários do $\mathrm{PBF}^{8,29}$. Hipóteses têm sido descritas ${ }^{30-32}$ como responsáveis pelo excesso de peso em populações vulneráveis, entre elas estão as alterações metabólicas decorrentes da precária condição nutricional vivenciadas na infância, onde acarreta-se um balanço energético positivo e o ganho de peso na vida adulta, além do consumo de alimentos ricos em calorias e alto índice glicêmico, escassos em nutrientes, que são comumente consumidos nestas populações. Pode-se dizer que a insegurança alimentar e à obesidade apresentam uma associação paradoxal, com o aumento de gordura sendo uma resposta fisiológica a I $\mathrm{A}^{33}$. 
Palmeira das Missões está localizada em uma região onde a monocultura e o latifúndio pelo cultivo de soja predominam com intensidade. A monocultura caracterizase pelo cultivo hegemônico de uma espécie agrícola, em uma determinada área ou região, ocorre normalmente em grandes propriedades rurais que visam a exportação ${ }^{34}$. A imposição da monocultura apresenta forte custo ambiental, social e cultural, pois pode haver um aumento da pobreza nas regiões de latifúndios por conta da concentração de renda. Os resultados encontrados na presente pesquisa podem ser apontados como um reflexo causado por este manejo econômico no município ${ }^{35}$.

Os dados apresentados demonstram a necessidade de recebimento e implementação de PTCR em famílias vulneráveis. Neste estudo, grande parte das mulheres pertencem ao quadro de famílias na classe social D-E, porém, um pequeno percentual pertence às classes $\mathrm{C} 1$ e B2, isso pode ser ao fato de que o questionário utilizado para avaliar a classe social, avalia aos bens que a família possui, como móveis, eletrodomésticos e habitação, não avaliando a renda mensal ou per capita bruta, e ainda o benéfico também visa a melhora na qualidade de vida destas famílias e a aquisição bens pode ser o reflexo desta ${ }^{27}$. Outro fator que deve ser mencionado é a ocorrência do desvio da finalidade do programa.

O presente estudo encontrou algumas limitações, entre elas, a não avaliação do nível de atividade física e dados sobre a ocupação das mulheres, tendo em vista que estes podem ser outros determinantes para a elevada prevalência de excesso de peso; e a utilização do formulário do SISVAN isoladamente como marcador do consumo alimentar. Além disso, quando se avalia a insegurança alimentar associada a renda e ao consumo alimentar, outras variáveis devem ser estudadas a fim de que se tenha maior compreensão dos fatores empíricos relacionadas a ela; A condição de vida destas mulheres (avaliada por outros indicadores), a percepção e conhecimento a cerca de uma alimentação saudável, acesso aos alimentos.

Mensurar a insegurança alimentar é complexo, pois existem múltiplos fatores presentes, assim nenhum indicador isolado consegue abranger sua dimensão. Diante dos resultados apresentados no estudo, observa-se uma alta prevalência insegurança alimentar ligada diretamente aos fatores socioeconômicos. Quase metade desta população vive em restrições quantitativas (IA moderada e grave), e a outra parcela em restrições qualitativas (IA leve), os níveis de sobrepeso e obesidade são alarmantes, a baixa escolaridade dessas mulheres e má qualidade da alimentação presente, demonstram o grave problema de saúde pública. Desta forma, os gestores das mais diferentes esferas, devem promover e implementar/implantar políticas intersetoriais e equitativas que garantam a esta população, a realização do direito humano a alimentação de forma contemplar, objetivando a redução da desigualdade, a fim de promover a segurança alimentar da população, visando estratégias com promoção da saúde e redução de peso.

\section{REFERÊNCIAS}

1. Silva JG, Grossi MED, França CG (org.). Fome zero: a experiência brasileira. Brasília: MDA; 2010.

2. Brasil. Lei no 11.346 , de 15 de setembro de 2006. Cria o sistema nacional de segurança alimentar e nutricional - SISAN com vistas em assegurar o direito humano à alimentação adequada e dá outras providências [Internet]. Brasília: Presidência da República; 2010 [capturado em 12 nov. 2019]. Disponível em: http://www. planalto.gov.br/ccivil_03/_Ato2004-2006/2006/Lei/L11346.htm

3. FAO. The state of food insecurity in the world: meeting the 2015 international hunger targets: taking stock of uneven progress. Rome: FAO; 2015. https://doi.org/10.3945/an.115.009936

4. Brasil. Decreto no 5.209, de 17 de setembro de 2004. Regulamenta a Lei no 10.836, de 9 de janeiro de 2004, que cria o programa Bolsa Família, e dá outras providências [Internet]. Brasília: Presidência da República; 2004 [capturado em 13 nov. 2019]. Disponível em: http://www.planalto.gov.br/ccivil_03/_ Ato2004-2006/2004/Decreto/D5209.htm

5. Brasil. Lei no 10.836, de 9 de janeiro de 2004. Cria o programa Bolsa Família e dá outras providências [Internet]. Brasília: Presidência da República; 2004 [capturado em 13 nov. 2019]. Disponível em: http://www.planalto.gov.br/ccivil_03/_Ato20042006/2004/Lei/L10.836.htm

6. Cabral CS, Lopes AG, Lopes JM, Vianna RPT. Food security, income, and the Bolsa Família program: a cohort study of municipalities in Paraíba State, Brazil, 2005-2011. Cad Saúde Pública. 2014;30(2):393-402. http://dx.doi.org/10.1590/0102$311 \times 00140112$

7. Instituto Brasileiro de Análises Sociais e Econômicas. Repercussões do programa Bolsa Família na segurança alimentar e nutricional das famílias beneficiadas. Rio de Janeiro: IBASE; 2008. https://doi. org/10.20435/inter.v19i3.1636

8. Cabral MJ, Vieira KA, Samaya AL, Florêncio TMMT. Perfil socioeconômico, nutricional e de ingestão alimentar de beneficiários do Programa Bolsa Família. Estud Av. 2013;27(78):71-87. http://dx.doi.org/10.1590/S010340142013000200006

9. Lima FEL, Fisberg RM, Uchimura KY, Picheth T. Programa BolsaFamília: qualidade da dieta de população adulta do município de Curitiba, PR. Rev Bras Epidemiol. 2013;16(1):58-67. http://dx.doi. org/10.1590/S1415-790X2013000100006

10. Monteiro F, Schmidt ST, Costa IB, Ameida CCB, Matuda NS. Bolsa Família: insegurança alimentar e nutricional de crianças menores de cinco anos. Ciênc Saúde Coletiva. 2014;19(5):1347-57. http:// dx.doi.org/10.1590/1413-81232014195.21462013 
11. Souza NN, Dias MM, Sperandio N, Franceschini SCC, Priore SE. Perfil socioeconômico e insegurança alimentar e nutricional de famílias beneficiárias do Programa Bolsa Família no município de Viçosa, Estado de Minas Gerais, Brasil, em 2011: um estudo epidemiológico transversal. Epidemiol Serv Saúde. 2012;21(4):655-62. http://dx.doi.org/10.5123/S167949742012000400015

12. Sperandio N, Priore SE. Prevalência de insegurança alimentar domiciliar e fatores associados em famílias com pré-escolares, beneficiárias do Programa Bolsa Família em Viçosa, Minas Gerais, Brasil. Epidemiol Serv Saúde. 2015;24(4)739-48. http://dx.doi. org/10.5123/S1679-49742015000400016

13. Deepask. Soja: Veja produção agrícola e área plantada por cidade do Brasil [Internet]. Palmeira das Missões: Deepask; 2011 [capturado em 10 jan. 2017]. Disponível em: http://www. deepask.com/goes?page=Soja:-Veja-a-producao-agricola-e-a-areaplantada-no-seu-municipio

14. Instituto Brasileiro de Geografia e Estatística. Dados estatísticos provenientes do censo demográfico. Rio de Janeiro: IBGE; 2010.

15. Brasil. Ministério do Desenvolvimento Social e Combate à Fome. Relatórios de informações sociais. Brasília: MDS; 2016.

16. Instituto Brasileiro de Geografia e Estatística. Pesquisa nacional por amostras de domicílios: segurança alimentar 2013. Brasília: IBGE; 2013.

17. Instituto Brasileiro de Geografia e Estatística. Pesquisa suplementar de segurança alimentar PNAD 2013: a percepção das famílias em relação ao acesso aos alimentos. Rio de Janeiro: IBGE; 2014. https://doi.org/10.17143/ciaed/xxiilciaed.2017.00322

18. Brasil. Ministério da Saúde. Secretaria de Atenção à Saúde. Departamento de Atenção Básica. Orientações para avaliação de marcadores de consumo alimentar na atenção básica. Brasília: MS; 2015. https://doi.org/10.11606/d.7.2012.tde-20022013153000

19. Associação Brasileira de Empresas de Pesquisa. Critério de classificação econômica Brasil. São Paulo: ABEP; 2015.

20. Brasil. Ministério da Saúde. Secretaria de Atenção à Saúde. Departamento de Atenção Básico. Orientações para a coleta e análise de dados antropométricos em serviços de saúde: Norma Técnica do Sistema de Vigilância Alimentar e Nutricional - SISVAN. Brasília: MS; 2011. https://doi.org/10.5123/s167949742019000100016

21. Brasil. Ministério da Saúde. Centro Brasileiro de Análise e Planejamento. Pesquisa Nacional de Demografia e Saúde da Criança e da Mulher: PNDS 2006: dimensões do processo e produtivo e da saúde da criança. Brasília: MS; 2009. https://doi. org/10.21115/jbes.v11.n2.p112-8

22. Uchimura KY, Bosi MLM, Lima FEL, Dobrykopf VF. Qualidade da alimentação: percepções de participantes do programa bolsa família. Ciênc Saúde Coletiva. 2012;17(3):687-94. http://dx.doi. org/10.1590/S1413-81232012000300015
23. Morais DC, Dutra LV, Franceschini SCC, Priore SE. Insegurança alimentar e indicadores antropométricos, dietéticos e sociais em estudos brasileiros: uma revisão sistemática. Ciênc Saúde Coletiva. 2014;19(5):1475-88. http://dx.doi.org/10.1590/141381232014195.13012013

24. Champagne CM, Casey PH, Connell CL, Stuff JE, Gossett JM, Harsha DW, McCabe-Sellers B, Robbins JM, Simpson PM, Weber JL, Bogle ML; Lower Mississippi Delta Nutrition Intervention Research Initiative. Poverty and food intake in rural America: diet quality is lower in food insecure adults in the Mississipi Delta. J Am Diet Assoc. 2007;107(11):1886-94. http://dx.doi.org/10.1016/j. jada.2007.08.003

25. Ruschel LF, Henn RL, Backes V, Melo P, Marques LAS, Olinto MTA. Insegurança alimentar e consumo alimentar inadequado em escolares da rede municipal de São Leopoldo, RS, Brasil. Ciênc Saúde Coletiva. 2016;21(7):2275-85. http://dx.doi. org/10.1590/1413-81232015217.00742015

26. Panigassil G, Segall-Corrêal NA, Marin-Leónl L, Pérez-Escamillall R, Maranhal LK, Sampaiol MFA. Intra-family food insecurity and profile of food consumption. Rev Nutr. 2008;21(suppl): 135s-44s. http://dx.doi.org/10.1590/S1415-527320080007 00012

27. Nascimento EC, Cruz BEV, Calvi MF, Carvalho JPL, Reis CC, Gomes DL. A influência do Programa Bolsa Família nas práticas alimentares das famílias do Território do Marajó, Pará, Brasil. Scientia Plena. 2016;12(6):1-11. http://dx.doi.org/10.14808/sci. plena.2016.069931

28. Brasil. Ministério da Saúde. Secretaria de Atenção à Saúde. Departamento de Atenção à Saúde. Guia alimentar para a população brasileira. 2. ed. Brasília: Ministério da Saúde; 2014. https://doi.org/10.11606/t.7.2009.tde-11012010145632

29. Lima FEL, Rabito El, Dias MRMG. Estado nutricional de população adulta beneficiária do Programa Bolsa Família no município de Curitiba, PR. Rev Bras Epidemiol. 2011;14(2): 198-206. http://dx.doi.org/10.1590/S1415-790X20110002 00002

30. Monteiro CA, Conde WL, Popkin BM. Independent effects of income and education on the risk of obesity in the Brazilian adult population. J Nutr. 2001;131(3):881S-6S. https://doi.org/10.1093/ jn/131.3.881s

31. Drenowsky A. Obesity, diets and social inequality. Nutr Rer. 2009;67 Auppl 1:36-9. http://dx.doi.org/10.1111/j.1753-4887. 2009.00157.x

32. Hruschka DJ. Do economic constraints on food choice make people fat? A critical review of two hypotheses for the povertyobesity paradox. Am J Hum Biol. 2012;24(3):277-85. https://doi. org/10.1002/ajhb.22231

33. Dhurandhar EJ. The food-insecurity obesity paradox: a resource scarcity hypothesis. Physiol Behav. 2016;162:88-92. https://doi. org/10.1016/j.physbeh.2016.04.025 
34. Brauner MCC, Graff L. Segurança alimentar e produção agrícola: reflexões sob a ótica da justiça ambiental. Veredas do Direito. 2015;12(24):375-400. http://dx.doi.org/10.18623/rvd.v12i24. 280

35. Alcantara Filho JL, Fontes RMO. A formação da propriedade e a concentração de terras no Brasil. História Econômica \& Economia Regional Aplicada. 2009;4(7)63-85.

\section{Autoras:}

JAQUIELI GRUHM FRANCO

Nutricionista graduada pela Universidade Federal de Santa Maria (UFSM), Campus

Palmeira das Missões, Palmeira das Missões, RS, Brasil.

Orcid: https://orcid.org/0000-0002-4315-8989

E-mail: jaquielifranco@gmail.com

MARILENE CASSEL BUENO

Nutricionista. Mestranda no Programa de Pós-Graduação em Desenvolvimento Rural,

Universidade Federal do Rio Grande do Sul, Porto Alegre, RS, Brasil.

Orcid: https://orcid.org/0000-0001-9229-519X

E-mail: marilenecassel@outlook.com

VANESSA RAMOS KIRSTEN

Nutricionista. Doutora pelo Programa de Pós-Graduação em Saúde da Criança e do Adolescente. Professora do Departamento de Alimentos e Nutrição e do Programa de Pós-Graduação em Gerontologia da UFSM, Campus Palmeira das Missões, Palmeira das Missões, RS, Brasil.

Orcid: https://orcid.org/0000-0002-6737-1039

E-mail: kirsten.vr@gmail.com

GREISSE Viero dA SiLVA LEAL

Nutricionista. Doutora em Saúde Pública pela Universidade de São Paulo. Professora do Departamento de Alimentos e Nutrição e do Programa de Pós-Graduação em Gerontologia da UFSM, Campus Palmeira das Missões, Palmeira das Missões, RS,

Brasil

Orcid: https://orcid.org/0000-0002-1204-0080

E-mail: greisse@hotmail.com 